\section{BMJ Paediatrics Open}

\title{
Associations between birth at, or after, 41 weeks gestation and perinatal encephalopathy: a cohort study
}

David E Odd, ${ }^{1,2,3}$ Christopher Yau, ${ }^{1,3}$ Cathy Winter, ${ }^{1,3}$ Timothy Draycott, ${ }^{1,3}$ Finn Rasmussen ${ }^{4}$

To cite: Odd DE, Yau C, Winter C, et al. Associations between birth at, or after, 41 weeks gestation and perinatal encephalopathy: a cohort study. BMJ Paediatrics Open 2018;2:e000010. doi:10.1136/ bmjpo-2017-000010

- Additional material is published online only. To view please visit the journal online (http://dx.doi.org/10.1136/ bmjpo-2017-000010).

Received 16 March 2017 Revised 16 November 2017 Accepted 12 December 2017
Check for updates

${ }^{1}$ School of Social and Community Medicine, University of Bristol, Bristol, UK ${ }^{2} \mathrm{NIHR}$ Bristol Biomedical Research Centre, University of Bristol, Bristol, UK

${ }^{3}$ Women and Children's Health, North Bristol NHS Trust, Bristol, UK

${ }^{4}$ Department of Public Health Sciences, Karolinska Institutet, Stockholm, Sweden

Correspondence to Dr David E Odd; david.odd@ bristol.ac.uk

\section{ABSTRACT}

Background Preterm birth causes long-term problems, even for infants born 1 or 2 weeks early. However, less is known about infants born after their due date and over a quarter of infants are born over 1 week late, and many still remain undelivered after 2 weeks. The aim of this work is to quantify the risks of infants developing encephalopathy when birth occurs after the due date, and if other proposed risk factors modify this relationship.

Methods The dataset contain information on 4036346 infants born in Sweden between 1973 and 2012. Exposure was defined as birth 7 , or more, days after the infants' due date. The primary outcome was the development of neonatal encephalopathy (defined as seizures, encephalopathy or brain injury caused by asphyxia or with unspecified cause). Covariates were selected as presumed confounders a priori.

Results $28.4 \%$ infants were born 1 or more weeks after their due date. An infant's risk of being born with encephalopathy was higher in the post 41 weeks group in the unadjusted (OR $1.40(95 \% \mathrm{Cl} 1.32$ to 1.49$)$ ) and final model (OR $1.38(95 \% \mathrm{Cl} 1.29$ to 1.47$)$ ), with the relative odds of encephalopathy increasing by an estimated $20 \%$ per week after the due date, and modified by maternal age $(\mathrm{P}=0.022)$

Conclusions Singleton infants born at, or after, 41 weeks gestation have lower Apgar scores and higher risk of developing encephalopathy in the newborn period, and the association appeared more marked in older mothers. These data could be useful if provided to women as part of their decision-making.

\section{INTRODUCTION}

Preterm birth causes long-term problems, even for infants born 1 or 2 weeks early. However, less is known about infants born after their due date and over a quarter of infants are born over 1 week late, and many still remain undelivered after 2 weeks. While there is a plethora of work investigating the long-term cognitive, educational and social functioning of babies born prematurely, ${ }^{12}$ little data exist for the outcomes of those infants born after their due date or the mechanisms of any possible adverse outcomes. ${ }^{3}$

\section{What is already known on this topic?}

- Preterm birth causes long-term problems, but less is known about infants born after their due date.

- Perinatal asphyxia may increase as infants pass their due date, but estimates are conflicting and imprecise.

- A current research priority is to try to identify babies at particularly high risk of morbidity and mortality who will benefit from induction.

\section{What this study hopes to add?}

The risks of developing perinatal encephalopathy increase among infants that remain in utero after their due date.

- The association was observed in the most recent data, in younger and older mothers and in wellgrown infants.

- However, this increase in risk is higher in women over 35 years old.

Perinatal asphyxia (deprivation of oxygen during birth) is a relatively common event with around $7 \%$ of infants needing support after birth. ${ }^{4}$ While most infants tolerate it well, a small proportion, around $0.2 \%{ }^{4}$ of these develop immediate signs of brain damage (hypoxic-ischaemic encephalopathy). ${ }^{5}$ The clinical impacts can be lifelong and devastating for them and their family; the litigation and societal costs notwithstanding. ${ }^{6}$ The risks of perinatal asphyxia may increase in infants born after their due date, ${ }^{3}$ but estimates are conflicting. ${ }^{7}$ However, in contrast to preterm birth, interventions can be used to deliver the infant if the risks of continuing the pregnancy are higher than delivery for either the mother or the infant. Indeed, induction of labour (IOL) has been shown to be beneficial in high-risk groups ${ }^{8-11}$ but its place in the management of infants past their due date is unclear. $^{11} 12$ 
This dearth of data makes counselling for women difficult and consequently the management of pregnancies beyond term varies hugely between units and countries (eg, $0.4 \%$ in Austria vs $7 \%$ in Denmark).$^{13}$ Current National Institute for Health and Clinical Excellence guidelines suggest that a research priority is to identify babies at particularly high risk of morbidity and mortality who will benefit from induction and therefore avoid induction for babies who do not need it ${ }^{14}$ - the primary aim of this work. However, even small improvements in outcomes would yield substantial health benefits for individuals and economic benefits for health services and society.

The aim of this work is to quantify the risks of developing perinatal encephalopathy or being born with low Apgar score (less than 7 at $5 \mathrm{~min}$ ), when born 7 or more days after their due date (at, or after, 41 completed weeks of gestation).

\section{METHODS}

\section{Population}

The dataset reviewed contains information on 4036346 infants born in Sweden between 1973 and 2012 and registered on the nationwide birth registry of Sweden. The birth registry provides data on $98 \%-99 \%$ of births and includes Apgar scores, as well as both neonatal and maternal diagnoses (coded using the International Classification of Diseases (ICD), 8th, 9th and 10th revisions). Details of the clinical signs that led to the diagnoses are not recorded.

Infants born before 37 weeks gestation ( $n=234924)$, from multiple births ( $\mathrm{n}=56493$ ) or with cardiovascular, respiratory, neurological or multiple system congenital abnormalities $(n=29535)$ were removed, leaving 3715394 infants for the study. Infants with missing data on Apgar score or gestational age $(n=7715)$ and those with missing data on at least one covariate $(n=280229)$ were also removed, leaving 3427450 subjects available for the analyses (92\% of eligible subjects) (online supplementary appendix 1).

Information on birth condition and perinatal wellbeing was retrieved from the birth registry. The exposure was defined as birth 7 , or more, days after the infants' due date ( $\geq 41$ weeks gestation). A primary outcome was the development of likely perinatal encephalopathy (defined as an ICD coding for either 'seizures', 'encephalopathy' or brain injury caused by 'asphyxia' or with 'unspecified cause' immediately after birth). ${ }^{15}$ Secondary outcomes were Apgar scores at 1,5 and $10 \mathrm{~min}$, or a low Apgar score at 5 min (less than 7$){ }^{16}$

\section{Potential confounders and covariates}

Covariates were selected as presumed confounders a priori, ${ }^{17}$ and categorised into three groups: (1) social (maternal age, maternal occupation, maternal education), (2) antenatal (infant gender and primiparity) and (3) intrapartum and potential causal factors (maternal or infant infection, birth weight (gram), birth by caesarean section).

\section{Statistical analysis}

Initially, subjects with and without missing data were compared. The distribution of risk factors and potential confounders was investigated between the infants split by their gestation at birth. In univariable analysis the mean Apgar score at 1, 5 and $10 \mathrm{~min}$, and the risk of developing encephalopathy was derived, split by each completed week of gestation. Due to the non-normal distribution of the Apgar score, geometric means (with 95\% CIs) were derived. Birth weight and maternal age were treated as continuous variable and not grouped further beyond their initial units (grams and years, respectively).

In the multivariable analyses, linear (for Apgar scores) and logistic regression (for encephalopathy and a low Apgar score at $5 \mathrm{~min}$ ) models were used to assess any association with gestational age at birth. To account for changes in coding over time, models were multilevel to account for year of birth. Adjustment for social and antenatal confounders was performed by adding the variables described above to the models in blocks of common variables (defined above). Variables with potential confounding influence, but with possible causal inference, were introduced in a final model. The impact of birth at $\geq 41$ weeks gestation on encephalopathy was calculated using the population attributable risk from this model.

Five sensitivity analyses were performed:

1. The model was repeated using the number of weeks of birth after the due date as an ordinal variable.

2. The model was repeated limiting the analysis to well grown (over $2500 \mathrm{~g}$ at birth) infants.

3 . The model was repeated, testing if maternal age (greater than 35 years of age), interacted with the association between birth $\geq 41$ weeks gestation and risk of encephalopathy.

4. The model was repeated, restricting the analysis to the most recent 10 years of data (2003-2012).

5. The model was repeated using an imputation technique (multiple imputation using chained equations) to impute missing covariate values. Exposure and outcome data (eg, encephalopathy or gestational age data) was not imputed. Because of computation limitations, only a random $10 \%$ of infants without encephalopathy were included in this analysis to maintain a practical sized dataset, and the model weighted to represent the initial population (20 datasets with 42102 infants in each).

All analyses were conducted with Stata V.10.0 software (StataCorp), and results are presented as number (per cent), mean (SD), OR (95\% CI) or median (IQR) as appropriate. Due to using registry data, informed consent was not obtained, and all data were provided and analysed anonymously. 


\section{RESULTS}

Infants with missing data weighed less and had older mothers (online supplementary appendix 2). Infants excluded with missing data were likely to have missing data for multiple variables. They were less likely to be exposed to pre-eclampsia, but more likely to have been exposed to maternal or neonatal infection. Mothers were more likely to have been nulliparous, and had a different profile of occupations and educational achievements (all comparisons $\mathrm{P}<0.001)$. There was no difference in gender $(\mathrm{P}=0.726)$ or risk of encephalopathy $(\mathrm{P}=0.136)$ between those with or without complete data.

In total, 973430 (28.4\%) infants were born 1 or more weeks after their due date. Over the 40-year period, there was a slight reduction in the (geometric) mean in the population from $40.1(40.0-40.1)$ in 1973 to 39.6 (39.6-39.6) $(\mathrm{P}<0.001)$ in 2012. Populations were similar, although infants born $\geq 41$ weeks gestation were more likely to be male, had greater birth weight and were less likely to be exposed to maternal pre-eclampsia, or be born by caesarean section (table 1). They were also more likely to develop maternal or neonatal infection. Mothers were younger, more likely to be nulliparous, and had a different profile of occupations and educational qualifications (all comparisons $\mathrm{P}<0.001$ ). Due to the large numbers in the dataset differences were often small and of questionable clinical relevance.
There was strong evidence that the mean Apgar score at 1,5 and $10 \mathrm{~min}$ reduced as the infants gestational age continued beyond 39 weeks (figure 1 and table 2, all $\mathrm{P}<0.001$ ), although the magnitude of the difference in mean values was small. In addition, the risk of being born with a low Apgar score and the risk of developing encephalopathy became greater as the infants gestational age increased (both $\mathrm{P}<0.001$ ). Interestingly, infants born at 37 weeks also appeared to have lower Apgar scores and higher rates of encephalopathy than infants born at 39 or 40 weeks gestation (post hoc analysis; $\mathrm{P}<0.001$ ). Infants born at 45 weeks gestation appeared to have the lowest rate of encephalopathy, however, this is based on only one infant developing encephalopathy and hence the CIs are wide around the point estimate $(0.07 \%(95 \%$ CI $-0.07 \%$ to $0.20 \%)$ ) and this observation needs to be interpreted with caution.

Overall, infants born $\geq 41$ weeks gestation were more likely to develop encephalopathy than those born before 41 weeks gestation $(3040(0.12 \%)$ vs $1640(0.17 \%)$, $\mathrm{P}<0.001)$. In the unadjusted results, the mean Apgar scores at 1,5 and $10 \mathrm{~min}$ were lower in infants born $\geq 41$ weeks gestation and this persisted after adjustment for a priori defined confounders, and remained after adjusting for both confounders and other causal factors (table 3). The risk of encephalopathy showed a similar association, with an infant's risk of being born with encephalopathy

\begin{tabular}{|c|c|c|c|}
\hline Measure & $37+0$ to $40+6$ weeks & $41+0$ and above & Difference $(95 \% \mathrm{Cl})$ \\
\hline \multicolumn{4}{|l|}{ Antenatal factors } \\
\hline Male & $1244878(50.7 \%)$ & $511567(52.6 \%)$ & $1.82 \%(1.71 \%$ to $1.94 \%)$ \\
\hline Birth weight (g) & $3511(473)$ & $3798(474)$ & 288 (287 to 289 ) \\
\hline Maternal pre-eclampsia & $115578(4.7 \%)$ & $38693(4.0 \%)$ & $-0.07 \%(-0.08 \%$ to $0.07 \%)$ \\
\hline \multicolumn{4}{|l|}{ Intrapartum factors } \\
\hline Maternal infection & $16092(0.66 \%)$ & $7240(0.74 \%)$ & $0.09 \%(0.07 \%$ to $0.11 \%)$ \\
\hline Neonatal infection & $10334(0.4 \%)$ & $6125(0.6 \%)$ & $0.21 \%(0.19 \%$ to $0.22 \%)$ \\
\hline Caesarean section & 294707 (12.1\%) & $85400(8.8 \%)$ & $-3.24 \%(-3.31 \%$ to $-3.16 \%)$ \\
\hline \multicolumn{4}{|l|}{ Demographic factors } \\
\hline Maternal age & $28.1(5.2)$ & $27.8(5.1)$ & $-0.30(-0.31$ to -0.29$)$ \\
\hline Primiparae & $995171(40.6 \%)$ & $450385(46.3 \%)$ & $5.72 \%(5.60 \%$ to $5.83 \%)$ \\
\hline \multicolumn{4}{|l|}{ Maternal occupation } \\
\hline Manual & $793546(32.3 \%)$ & $314211(32.3 \%)$ & $-0.06 \%(-0.17 \%$ to $0.05 \%)$ \\
\hline Non-manual & $868071(35.4 \%)$ & $371740(38.2 \%)$ & $2.82 \%$ (2.70\% to $2.93 \%)$ \\
\hline Other & $792403(32.3 \%)$ & $287479(29.5 \%)$ & $-2.86 \%(-2.87 \%$ to $-2.65 \%)$ \\
\hline \multicolumn{4}{|l|}{ Maternal education status } \\
\hline$<9$ years & $71148(2.9 \%)$ & $28652(2.9 \%)$ & $0.04 \%(0.04 \%$ to $0.08 \%)$ \\
\hline $9-10$ years & $919214(37.5 \%)$ & $364418(37.4 \%)$ & $-0.02 \%(-0.01 \%$ to $0.09 \%)$ \\
\hline Full secondary & $465920(19.0 \%)$ & $177561(18.2 \%)$ & $-0.75 \%(-0.84 \%$ to $-0.65 \%)$ \\
\hline Higher education & 997738 (40.7\%) & 402799 (41.4\%) & $0.72 \%$ (0.61\% to $0.84 \%)$ \\
\hline
\end{tabular}

Values are number (per cent), mean $( \pm \mathrm{SD})$ or difference $(95 \% \mathrm{Cl})$ as appropriate. 


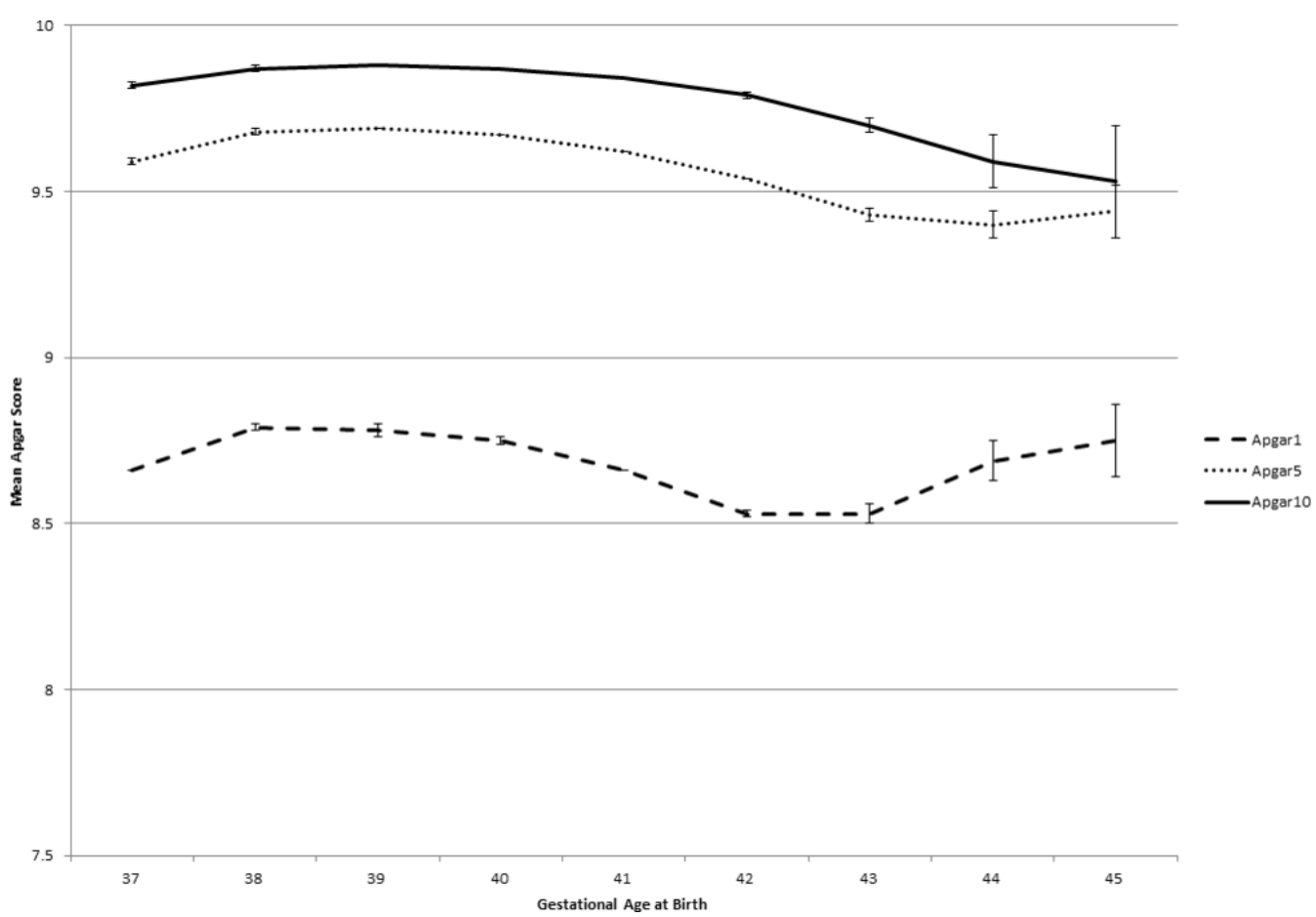

Figure 1 Mean Apgar scores, split by gestational age at birth.

higher if they were born 1 or more weeks after their due date, in the unadjusted (OR 1.40 (95\% CI 1.32 to 1.49)) and adjusted analysis (OR 1.34 (95\% CI 1.26 to 1.42)), and also persisted in the model controlling for potential causal factors (OR 1.38 (95\% CI 1.29 to 1.47$)$ ). The risk of a low $(<7) 5$ min Apgar score showed similar results (all comparisons; $\mathrm{P}<0.001$ ). The population attributable risk fraction of infants who developed encephalopathy at, or after, 41 weeks gestation was $4.6 \%$.

Using birth after the due date as an ordinal variable (eg, 1 week over at 41 weeks, 2 weeks at 42 weeks, etc) demonstrated similar results to the main analysis: for each week after the due date that the infant stayed in utero, there was an associated increase in the risk of developing neonatal encephalopathy (OR 1.22 (95\% CI 1.18 to 1.27$)$ ).

Repeating the model after restricting to well-grown infants (OR 1.36 (95\% CI 1.28 to 1.46)) or to the most recent 10 years of data (OR 1.35 (95\% CI 1.20 to 1.52)) provided very similar results.

There was evidence that maternal age modified the association between later birth and encephalopathy $(\mathrm{P}=0.022)$. Mothers aged 35 years and older had a higher risk that birth $\geq 41$ weeks would result in an infant with encephalopathy (OR 1.67 (95\% CI 1.40 to 1.98)) compared with those who were younger than 35 (OR 1.34 (95\% CI 1.25 to 1.43 )). A multiple imputation model including all infants with exposure and outcome date $(\mathrm{n}=717801$, adjusted OR $1.41 \quad(95 \%$ CI 1.31 to 1.51 )) also produced similar results to the main analysis. A final, post hoc analysis was performed due to the possible non-linear association of gestational age and encephalopathy. Infants were split by gestation age into three exposure groups; early (37/38 weeks), within 7 days of the due date $(39 / 40$ weeks $)$ and late $(\geq 41$ weeks). Infants born at 37/38 weeks (OR 1.30 (95\% CI 1.20 to 1.40 ) and $\geq 41$ weeks (OR 1.50 (95\% CI 1.40 to $1.60)$ ) both demonstrated higher risks of encephalopathy than those born within 7 days of their due date in the unadjusted analysis, but the effect was only seen in those $\geq 41$ weeks after correction for potential confounders (OR 1.01 (95\% CI 0.93 to 1.10) and OR 1.38 (95\% CI 1.29 to 1.48$)$, respectively).

\section{DISCUSSION}

We have demonstrated, in a large population-based dataset, that the risks of developing perinatal encephalopathy increases significantly for each week that pregnancies continue after their due date. Assuming that gestational age is accurately assessed and intrapartum care of pregnancies is similar for the gestational age at birth, then this effect may be readily preventable if this association is causal. Indeed, sensitivity analysis suggests that the relative risk of encephalopathy increases by an estimated $20 \%$ per week among infants that remain in utero after their due date (compared with infants born at, or before it). The association appeared robust, as it was observed in the most recent data, in younger and older mothers and in well-grown infants.

Limitations of this work include the use of routine data, and the number of infants excluded from the analysis due to missing data points. Likely perinatal encephalopathy was defined using available neonatal ICD codes consistent with our previous work, ${ }^{6}$ but may also include infants with other neurological disease, 
and does not identify if the infants were admitted to a neonatal intensive care unit (NICU) or not. As a routine data source, it is difficult to use the ICD codes to further interrogate the individual reasons for the neurological signs and some uncertainty about the underlying biological causes, and the assumption that it can be reversed by earlier delivery must remain. Equally, we did not have information on the indication for operative deliveries or IOL; although this is likely to cause us to underestimate any impact of postdue date delivery and this use of routine data is likely to weaken any association seen due to misclassification. However, the birth registry is considered a robust source of information, thus minimising any effect seen rather than introducing false positive findings. In particular, the measurement of gestational age in this cohort may be less accurate than modern measures available to clinicians and the methods used to derive it (initially clinical measure of last menstrual period, followed by ultrasound dating) have changed over the study period. However, while diagnostic criteria may have changed over the study period, the models used random effects to account in part for this, and results from the most recent years showed similar results to those from the whole cohort.

The groups of infants did appear to vary on a number of clinical factors (eg, infection risk), however, the cohort was large, and able to show differences in clinical characteristics (eg, a 2-3 months difference in maternal age) of minor magnitude and of questionable clinical significance. Overall, we saw little evidence for confounding in this work, but it should be noted that residual and uncontrolled confounding remain a limitation of any non-randomised study. With respect to missing data, we have attempted to investigate the impact using a multiple imputation technique, which produced almost identical results to the main analysis, although again, any interpretation of the results should consider this limitation.

This work suggests an increase in significant morbidity in infants born after more than 41 weeks gestation, and it seems plausible that the presumed deterioration in the in utero environment for the foetuses also contributes to the known risk of late stillbirth; possibly placental insufficiency. Alternatively we identified an increase in the risk of infection in the later-born infants $(0.6 \%$ vs $0.4 \%)$ which may have contributed to seizures or low Apgar scores, although this association persisted after adjustment for it in the final model. It should be noted that infants over 43 weeks gestation appeared to have slightly higher $1 \mathrm{~min}$ Apgar scores (but not 5 or $10 \mathrm{~min}$ ) than other post-term infants. Whether this was a statistical imprecision due to the low numbers in these groups or evidence that the score may be a less reliable measure of birth condition than the later measures is unclear.

It seems possible that IOL or caesarean section at 40 weeks gestation may improve morbidity and mortality, although we were unable to investigate perinatal deaths in 
Table 3 Regression models for the association between birth after $40+6$ weeks gestation with measures of birth condition

\begin{tabular}{|c|c|c|c|}
\hline \multirow[b]{2}{*}{ Measure } & Unadjusted & Adjusted* & Fully adjusted $\dagger$ \\
\hline & Mean difference $(95 \% \mathrm{Cl})$ & Mean difference $(95 \% \mathrm{Cl})$ & Mean difference $(95 \% \mathrm{Cl})$ \\
\hline \multicolumn{4}{|l|}{ Apgar score } \\
\hline $1 \min$ & $-0.13(-0.13$ to -0.13$)$ & $-0.12(-0.12$ to -0.11$)$ & $-0.12(-0.12$ to -0.11$)$ \\
\hline $5 \mathrm{~min}$ & $-0.05(-0.06$ to -0.05$)$ & $-0.05(-0.05$ to -0.05$)$ & $-0.05(-0.05$ to -0.05$)$ \\
\hline \multirow[t]{2}{*}{$10 \min$} & $-0.03(-0.03$ to -0.02$)$ & $-0.02(-0.02$ to -0.02$)$ & $-0.03(-0.03$ to -0.02$)$ \\
\hline & OR $(95 \% \mathrm{Cl})$ & OR $(95 \% \mathrm{Cl})$ & OR $(95 \% \mathrm{Cl})$ \\
\hline Low $(<7)$ Apgar score at $5 \mathrm{~min}$ & 1.36 (1.33 to 1.40$)$ & 1.31 (1.28 to 1.34$)$ & 1.45 (1.41 to 1.48$)$ \\
\hline Encephalopathy & $1.40(1.32$ to 1.49$)$ & 1.34 (1.26 to 1.42$)$ & $1.38(1.29$ to 1.47$)$ \\
\hline
\end{tabular}

Data are mean difference $(95 \% \mathrm{Cl})$ or OR $(95 \% \mathrm{Cl})$ for each increasing week of gestational age at birth.

All $P$ values are $<0.001$

${ }^{*}$ Adjusted for sex, parity, maternal age, maternal education and maternal occupation.

†Adjusted for maternal pre-eclampsia, multiple birth, caesarean section, birth weight, maternal infection and infant infection.

this work. However, early birth particularly by caesarean section is not without risk; in addition to respiratory morbidity, recent work suggests poorer long-term cognitive outcomes may occur for infants born just 2 weeks early, ${ }^{8}$ although we were unable to demonstrate a higher risk of encephalopathy in infants born before 38 weeks (after adjustment for confounders).

There is particular concern with regard to the risks associated with pregnancies continuing in older women, ${ }^{18}$ and this work demonstrates a higher risk in women over 35 years old. Furthermore, a recent interventional study for women aged over 35 years concluded that IOL at 39 weeks of gestation, as compared with expectant management, had no significant effect on the rate of caesarean section and no adverse short-term effects on maternal outcomes. However, that study was underpowered for neonatal outcomes. ${ }^{19}$ A recent systematic review demonstrating reductions in caesarean rates for women induced at term also showed that there was a reduced risk of fetal death and admission to NICUs with IOL. ${ }^{10}$

Despite these findings, there is no general consensus on whether IOL should be offered to all women at 40 weeks gestation. ${ }^{11}{ }^{12}$ Factors that should be considered before advocating such a recommendation would include the accuracy of the gestational age measurements, and impact of the induction process on a woman's birth experience. ${ }^{14}$ Indeed, midwives or obstetricians may be reticent to recommend an earlier intervention for risk of over medicalising normal pregnancies. There may also be implications on workload and capacity if women were to accept earlier IOL.

The results from this study provide further evidence to support elective IOL during the 39th or 40th week of gestation. However, more randomised trials of IOL at 40 weeks gestation versus expectant management need to be conducted to obtain prospective data on longterm neonatal and maternal outcomes. More research is required in this area, including work looking at the role of membrane sweeping or more formal induction processes, as well as quantifying any long-term consequences to the infant.

\section{CONCLUSION}

Singleton infants born at, or after, 41 weeks gestation have a higher risk of developing encephalopathy in the newborn period. Expediting birth at 40 weeks gestation, including IOL, could prevent a substantial proportion (up to 5\% (based on the estimated population attributable risk)) of all neonatal encephalopathy. These poor outcomes are more common the longer the pregnancy continues after the infants' due date and they do not appear to be restricted to older mothers or low birthweight infants. While these results should not be viewed in isolation, these data could usefully be provided to women as part of their decision-making with regard to IOL. Finally, further research is therefore needed to identify the pathways through which this effect is mediated, to ascertain appropriate intervention points and also establish the impact and risk/benefit ratio of pre-emptive birth.

Acknowledgements This study was supported by the NIHR Biomedical Research Centre at the University Hospitals Bristol NHS Foundation Trust and the University of Bristol. The views expressed in this publication are those of the author(s) and not necessarily those of the NHS, the National Institute for Health Research or the Department of Health.

Contributors DEO, CY, CW, TD and FR conceived the idea and developed the methodology. DEO performed the statistical analysis and wrote the first draft of the paper. DEO, CY, CW, TD and FR developed and approved the final manuscript.

Funding This research has been carried out through funding by the North Bristol NHS Trust Springboard Fund (Round 9, Ref: 13).

Competing interests None declared.

Ethics approval Ethical Review Board of Stockholm (Reference: 2015/1279-31/2) Provenance and peer review Not commissioned; externally peer reviewed.

Data sharing statement Data was obtained from Statistics Sweden (http://www. scb.se/en_/) and access is not avaliable from the authors.

Open Access This is an Open Access article distributed in accordance with the Creative Commons Attribution Non Commercial (CC BY-NC 4.0) license, which permits others to distribute, remix, adapt, build upon this work non-commercially, and license their derivative works on different terms, provided the original work is 
properly cited and the use is non-commercial. See: http://creativecommons.org/ licenses/by-nc/4.0/

(C) Article author(s) (or their employer(s) unless otherwise stated in the text of the article) 2018. All rights reserved. No commercial use is permitted unless otherwise expressly granted.

\section{REFERENCES}

1. Johnson S, Hennessy E, Smith R, et al. Academic attainment and special educational needs in extremely preterm children at 11 years of age: the EPICure study. Arch Dis Child Fetal Neonatal Ed 2009;94:F283-9.

2. Odd D, Evans D, Emond A. Preterm birth, age at school entry and educational performance. PLoS One 2013;8:e76615.

3. Moster D, Wilcox AJ, Vollset SE, et al. Cerebral palsy among term and postterm births. JAMA 2010;304:976-82.

4. Odd D, Lewis G, Whitelaw A, et al. Resuscitation at birth and cognition at 8 years of age: a cohort study. Lancet 2009;9:1615-22.

5. Azzopardi DV, Strohm B, Edwards AD, et al. Moderate hypothermia to treat perinatal asphyxial encephalopathy. $N$ Engl J Med 2009;361:1349-58.

6. Odd DE, Gunnell D, Lewis G, et al. Long-term impact of poor birth condition on social and economic outcomes in early adulthood. Pediatrics 2011;127:e1498-504.

7. Tita AT, Lai Y, Bloom SL, et al. Timing of delivery and pregnancy outcomes among laboring nulliparous women. Am J Obstet Gynecol 2012;206:239.e1-8.

8. Chiossi G, Lai Y, Landon MB, et al. Timing of delivery and adverse outcomes in term singleton repeat cesarean deliveries. Obstet Gynecol 2013;121:561-9.
9. Martinez-Biarge M, Madero R, González A, et al. Perinatal morbidity and risk of hypoxic-ischemic encephalopathy associated with intrapartum sentinel events. Am J Obstet Gynecol 2012;206:148. e1-7.

10. Mishanina E, Rogozinska E, Thatthi T, et al. Use of labour induction and risk of caesarean delivery: a systematic review and metaanalysis. Can Med Assoc J 2014;189:665-73.

11. Smith G. Labour should be induced at term: FOR: The balance of risks versus benefits favours offering term induction to all women. BJOG 2015;122:982

12. Jacquemyn Y. Labour should be induced at term: AGAINST: no proof of benefit. BJOG 2015;122:982.

13. Zeitlin J, Blondel $\mathrm{B}$, Alexander $\mathrm{S}$, et al. Variation in rates of postterm birth in Europe: reality or artefact? BJOG 2007;114:1097-103.

14. National Institute for Health and Clinical Excellence (NICE). Inducing labour (CG70). Manchester: NICE, 2008.

15. Odd DE, Lewis G, Whitelaw A, et al. Resuscitation at birth and cognition at 8 years of age: a cohort study. Lancet 2009;373:1615-22.

16. Committee on Obstetric Practice, ACOGAmerican Academy of PediatricsCommittee on Fetus and Newborn, ACOG. ACOG Committee Opinion. Number 333, May 2006 (replaces No. 174, July 1996): the Apgar score. Obstet Gynecol 2006;107:1209-12.

17. McNamee R. Confounding and confounders. Occup Environ Med 2003;60:227-34.

18. Walker KF, Bugg GJ, Macpherson M, et al. Induction of labour at term for women over 35 years old: a survey of the views of women and obstetricians. Eur J Obstet Gynecol Reprod Biol 2012:162:144-8.

19. Walker KF, Bugg GJ, Macpherson M, et al. Randomized trial of labor induction in women 35 years of age or older. $N$ Engl J Med 2016;374:813-22. 